[5] is conected at a point near the center of the first arm. The tension pulley [4] is actuated, together with the small roller [7] and two arms, by cam [9]. Cam shaft [8] is slowly rotated by the motor through a suitable speed-reducing device. If cam [9] is rotated from the place marked with the solid line to the place marked with the dotted line (see the figure), the operating diameter of variable pitch sheave [2] gets small, because variable pitch sheave is forced apart by V-belt [3]. The operating diameter of variable pitch sheave [2] can be reduced by increasing the rotational speed of drum-shaft [1].

If a cam of a suitable shape is used, the rotational speed of drum-shaft [1] increases or decreases suddenly, but the rotational speed of the package changes only gradually.

The time lag of the variation in the rotational speed of drum-shaft and package reduces the chances of the package becoming like entangled ribbons.

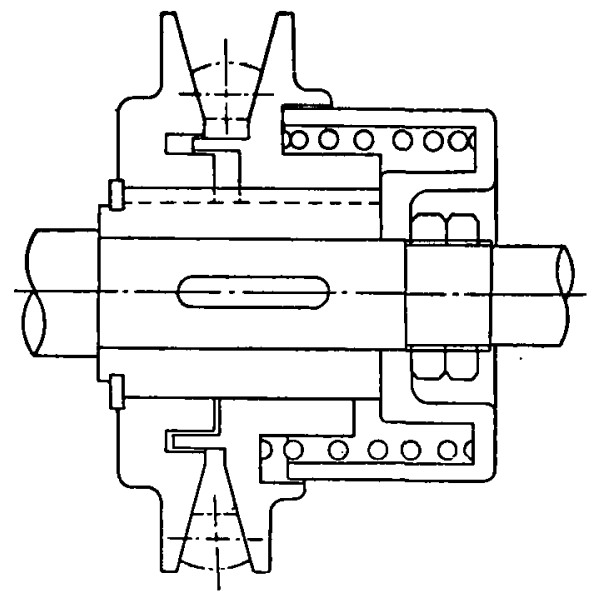

Fig. 2

\title{
Industrial Vacuum Cleaner for Textile Machines
}

\author{
Nippon Cleaner Co., Ltd.
}

Sangenjaya-machi, Setagaya-ku, Tokyo

Nippon Cleaner Co., Ltd. has perfected two types of industrial vacuum cleaners for textile machines. Type FA-31 is a cleaner for looms. Type FA-14 is for spinning machines, especially for fine spinning frames.

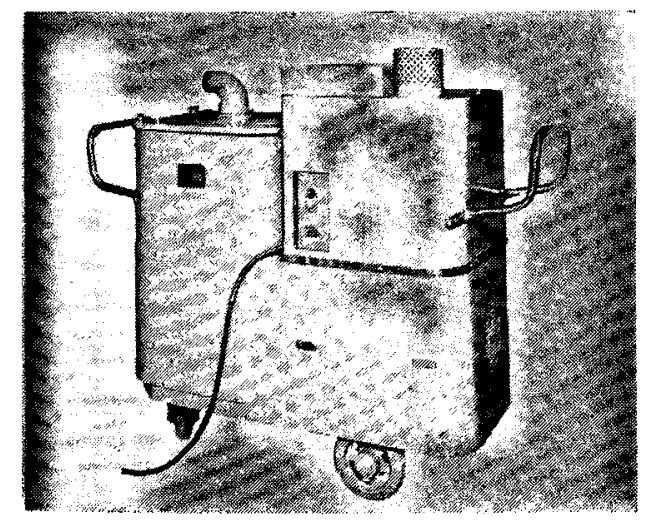

Fig. 2. Type FA-31 Vacuum Cleaner.

\section{Structures of the Industrial Vacuum Cleaners}

Each type of cleaner consists of a vacuum generator, a motor and a dust-sucking device complete with a filter. Figure 1 is a sketch of the structures of both types. Figure 2 is a photo of type FA-31.

\section{Feautures of the Industrial Vacuum Cleaners}

(I) Each type, equipped with an induction motor, can be worked for over 20 straight hours.

(2) Each type has a revolving wheel which is always free from dust (fibers, etc.).

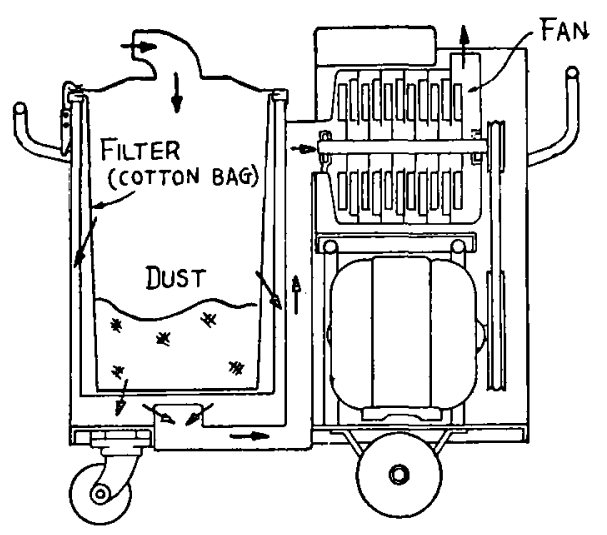

Fig. 1. Sketch of the structure of industrial vacuum cleaner.

(3) Each type is easy to use.

(4) Each type has a high-quality cotton bag, shaped like a bag, which sucks dust from its bottom. This means a large dust-sucking power. 
